\title{
Corticotropin-Releasing Factor Receptor 1 Activation During Exposure to Novelty Stress Protects Against Alzheimer's Disease-Like Cognitive Decline in A $\beta P P / P S 1$ Mice
}

\author{
Gillian A. Scullion ${ }^{\mathrm{a}}$, Katherine N. Hewitt ${ }^{\mathrm{a}, \mathrm{b}}$ and Marie-Christine Pardon ${ }^{\mathrm{a}, *}$ \\ a University of Nottingham Medical School, School of Biomedical Sciences, Neurodegeneration' group, \\ Queen's Medical Centre, Nottingham, UK \\ ${ }^{\mathrm{b}}$ Department of Experimental Psychology, University of Oxford, Oxford, UK
}

Handling Associate Editor: Laura Petrosini

Accepted 5 December 2012

\begin{abstract}
A lifestyle rich in physical and mental activities protects against Alzheimer's disease (AD) but the underlying mechanisms are unclear. We have proposed that this is mediated by a stress response and have shown that repeated exposure to novelty stress, which induces physical and exploratory activities, delays the progression of AD-like pathology in the TASTPM mouse model. Here, we aimed to establish the role played by corticotrophin-releasing factor receptor 1 (CRFR1), a major component of the stress axis, in TASTPM's behavioral and neuroendocrine responses to novelty and related protective effects. We show that the stress response of TASTPM mice is altered with reduced CRFR1-mediated neuroendocrine and behavioral responses to novelty and a distinct profile of behavioral responses. Repeated novelty-induced CRFR1 activation, however, mediated the improved contextual fear memory and extinction performance of TASTPM mice and increased hippocampal and fronto-cortical levels of synaptophysin, a marker of synaptic density, and fronto-cortical levels of the post-synaptic marker PSD95. The N-methyl-D-aspartate receptor (NMDAR) is the major receptor for synaptic plasticity underlying learning and memory. Although novelty-induced NMDAR activation contributed to enhancement of fear memory and synaptophysin levels, antagonism of CRFR1 and NMDAR prevented the novelty-induced increase in hippocampal synaptophysin levels but reversed the other effects of CRFR1 inactivation, i.e., the enhancement of contextual fear extinction and fronto-cortical synaptophysin and PSD95 levels. These findings suggest a novel mechanism whereby a stimulating environment can delay AD symptoms through CRFR1 activation, facilitating NMDAR-mediated synaptic plasticity and synaptogenesis in a region-dependent manner, either directly, or indirectly, by modulating PSD95.
\end{abstract}

Keywords: Active lifestyle, Alzheimer's disease, corticotrophin-releasing factor, genetic mouse model, learning and memory, synaptogenesis

Supplementary data available online: http://dx.doi.org/10.3233/JAD-122164

\section{INTRODUCTION}

${ }^{*}$ Correspondence to: Marie-Christine Pardon, $\mathrm{PhD}$, University of Nottingham Medical School, School of Biomedical Sciences, Neurodegeneration' group, Queen's Medical Centre, Nottingham, NG7 2UH, UK. Tel.: +44 11582 30149; Fax: +44 11582 30142; E-mail: marie.pardon@nottingham.ac.uk.
A number of studies have established that a lifestyle rich in physical and mental exercise decreases the risk and progression of Alzheimer's disease (AD) [1-3] but the underlying mechanisms are yet to be fully 
understood. Susceptibility to stress is a risk factor for AD but positive lifestyle factors effective in delaying $\mathrm{AD}$ progression in mouse models (e.g., exercise, environmental enrichment) improve stress resistance despite inducing a number of markers of chronic stress, such as a sustained activation of the hypothalamicpituitary-adrenal (HPA) axis $[1,4]$. This led us to the hypothesis that the protective effect on $\mathrm{AD}$ progression is mediated, at least in part, by a stress response.

In support of this hypothesis, we have shown that repeated exposure to novelty stress, induced by the placement of mice in a novel clean cage for repeated 1-hour sessions, induces HPA axis activation and locomotor and exploratory activities, which do not habituate with repeated exposure [5], while delaying the progression of contextual fear memory deficits and amyloid load in a genetic mouse model of AD (TASTPM mouse line) [4, 6]. Using this procedure and a pharmacological approach, we aimed to establish a role for corticotrophin-releasing factor receptor 1 (CRFR1) signaling in the cognitive benefits of novelty sessions in TASTPM mice.

Corticotrophin-releasing factor (CRF) is a major contributor to the stress response and is widely distributed within the central nervous system, including key areas affected by AD (e.g., hippocampus, cortex, and hypothalamus). CRF binds to two major receptors, CRFR1 and CRFR2, to mediate its effects, but CRFR1 is the most critical for stress responses and AD. In the hypothalamus, CRF controls the HPA stress response and subsequent release of glucocorticoids via CRFR1 [7]. CRF is also synthesized locally in response to stress in which it stimulates excitatory neurotransmission also via CRFR1, thereby mediating a range of functions necessary for adaptation [8] and relevant to $\mathrm{AD}$, such as cognition, neuroprotection, neuroinflammation, amyloid, and tau processing [9]. Postmortem observation of $\mathrm{AD}$ brains has revealed altered CRF and CRFR1 levels in the hypothalamus and other brain areas [9], together with increased circulating levels of glucocorticoids [10].

We therefore hypothesized that CRFR1 signaling would be reduced in TASTPM mice at a pre-pathological age contributing to altered stressresponses and disease progression. Accordingly, we characterized the behavioral and neuroendocrine responses to acute novelty stress and antagonism of the two CRF receptors, CRFR1 and CRFR2, in wild-type (WT) and TASTPM mice aged 4 months, when cognitive deficits and amyloid pathology are subtle [4]. We found that CRFR1-mediated effects are reduced in TASTPM mice and tested the hypothesis that this deficit would be rescued by repeated novelty sessions, contributing to the cognition-enhancing effects. The loss of synapses is the major correlate of cognitive decline in AD [11] and CRF, via CRFR1, modulates hippocampal synaptic function and plasticity and, thereby, learning and memory [12]. We, therefore, quantified hippocampal and fronto-cortical levels of the presynaptic protein synaptophysin and postsynaptic marker postsynaptic density protein 95 (PSD95). CRF modulates excitatory glutamatergic transmission in a CRF receptor-dependent manner [13] and the glutamatergic ionotropic N-methyl-D-aspartate receptor (NMDAR) is the major receptor for synaptic plasticity underlying learning and memory [14]. This prompted us to test the hypothesis that interactions between the two receptors contribute to the cognitionenhancing effects of repeated novelty. We found that, although CRFR1 signaling is reduced in prepathological TASTPM mice, its repeated activation mediates the cognitive and synaptic benefits of novelty sessions, but not via co-activation of NMDAR, except for enhancement of hippocampal synaptogenesis.

\section{MATERIAL AND METHODS}

\section{Animals}

TASTPM transgenic mice and wild type C57BL/6J control mice were generated and maintained as previously described [4, 6, 15,]. All procedures were undertaken in accordance with the UK animals Scientific Procedures Act under project license 40/2715.

\section{Study design}

Three experiments were performed. First, 4-monthold WT and TASTPM mice were either undisturbed or subjected to an acute novelty session ( $n=15$ /group). At the end of the 1-h session, trunk blood was collected for determination of plasma adrenocorticotropic hormone (ACTH) and corticosterone levels, their brain was collected, and the hippocampi and frontal cortices dissected on ice for assessment of glutamate levels ( $n=7$ /group) using high performance liquid chromatography or protein levels of the presynaptic marker synaptophysin and postsynaptic marker PSD95 ( $n=8$ /group) using western immunoblotting. This age is characterized by minor plaque pathology and subtle cognitive deficits $[4,16]$.

Because TASTPM mice differed from WT mice in their HPA response to novelty, and CRF receptors modulate HPA axis and behavioral reactivity 
to stress, the effects of antagonism of CRFR1 or CRFR2 on neuroendocrine, physiological, and behavioral responses to novelty stress were investigated ( $n=7-9$ per genotype and treatment condition). The number of fecal pellets and novelty-induced behaviors (rearing, leaning, grooming, and tail rattling) were recorded manually by an observer blind to the experimental groups as described previously [5].

Because TASTPM mice showed altered CRFR1mediated responses to novelty and because CRFR1 influences synaptic plasticity underlying learning and memory while and NMDAR is the major receptor for synaptic plasticity, we tested the hypothesis that both receptors interact to produce the cognition-enhancing effects of repeated exposure to novelty in TASTPM mice. Antagonists of CRFR1 and/or NMDAR were administered i.p. $30 \mathrm{~min}$ prior to each novelty session. After 5 weeks of exposure, mice were tested for locomotor, anxiety-related, and cognitive performance using the open-field and contextual fear conditioning test ( $n=10-13$ /group). $72 \mathrm{~h}$ after the last behavioral test, mice were killed by decapitation. Trunk blood was collected for assessment of plasma levels of ACTH and corticosterone, and their brain was removed and dissected for assessment of hippocampal and frontocortical levels of synaptophysin and PSD95. Because repeated injections are stressful, the impact of repeated injections was also tested in TASTPM and WT mice (8-10/group).

\section{Novelty stress}

The procedure was carried out as described previously $[4,17]$ either once for $1 \mathrm{~h}$ or 4 times $1 \mathrm{~h}$ /day for 5 weeks, by placing the mouse into a clean cage, devoid of food, water, or environmental enrichment and bisected by a Perspex partition in order to restrict the space available by half. Unexposed mice were handled.

\section{Drug treatments}

Because available CRF2 antagonists do not cross the blood-brain barrier, in the acute study, the CRFR1 antagonist CP154,526 (7 nmol; Tocris), CRFR2 antagonist anti-sauvagine (100 pmol; Bachem), or vehicle (saline) were administered i.c.v. by direct infusion into the right ventricle $(4 \mu \mathrm{l}$ for $5 \mathrm{~min}$, stereotaxic coordinates: AP $-0.3 \mathrm{~mm}$; $\mathrm{ML}+1 \mathrm{~mm}$ and $\mathrm{DV}-2.0 \mathrm{~mm}$; relative to bregma) under isofluorane anesthesia. The injector was then left in place for $1 \mathrm{~min}$, the incision was closed and the mouse left to recover for $30 \mathrm{~min}$, prior to being subjected to the acute novelty session and then, culled immediately for collection of trunk blood and verification of the injection site.

For the repeated exposure to novelty, the CRFR1 antagonist CP154,526 (10 mg/kg), NMDAR antagonist MK801 $(0.1 \mathrm{mg} / \mathrm{kg}$ ), or vehicle (saline $5 \%$ Tween-20) were administered i.p. $30 \mathrm{~min}$ before each session. The effect of a CRFR2 antagonist could not be tested due to animal welfare concerns, as it would require 20 i.c.v. injections under brief anesthesia.

\section{Behavioral assessment}

Locomotion in the open field test as well as acquisition, retention, and extinction of contextual fear conditioning were performed and analyzed as described previously $[4,18]$.

The open-field comprised a Perspex arena $(30 \times 35 \times 25 \mathrm{~cm})$ with transparent sides and a grey floor and behavior was recorded by a camera positioned directly above the arena. Locomotor activity (distance travelled) was automatically assessed using Ethovison Software (Noldus, Wageningen, Netherlands).

The shock chamber $(25 \times 25 \times 38 \mathrm{~cm})$ used for contextual fear conditioning comprised three stainless steel walls and one transparent Perspex wall. The floor consisted of stainless steel bars $1 \mathrm{~cm}$ apart that were connected to a shock generator (Campden Instruments, Loughborough, UK).

During the acquisition trial, mice received one $0.4 \mathrm{~mA}$ foot shock each minute for $10 \mathrm{~min}$ with the first shock given after $60 \mathrm{~s}$, resulting in a total of 10 shocks throughout the trial. A retention trial took place $24 \mathrm{~h}$ later in which the mice were placed back into the shock chamber for $3 \mathrm{~min}$ in the absence of shocks. $24 \mathrm{~h}$ after the retention trial, the mice were placed back in the chamber for a 3-min extinction trial, again in the absence of shocks. Again, activity was recorded and subsequently analyzed by an observer blind to genotype or treatment. Behavior was recorded by a camera positioned directly above the arena for subsequent analysis. Learning and memory were assessed by recording immobility levels during each trial. Immobility levels were scored by two observers blind to the experimental groups and averaged for statistical analyses. When the two values differed by more than $10 \mathrm{~s}$, the scoring was repeated and the two closest values used for analysis.

\section{Plasma hormone levels}

Trunk blood was separated as described previously [4]. Corticosterone and ACTH levels were assessed 
in duplicate using ELISAs (Stressgen and Phoenix Experimental, respectively) according to manufacturer's instructions.

\section{Western immunoblotting}

Tissue was lysed as described previously [19]. Lysates were normalized for total protein concentration using the BCA protein assay (Pierce). Proteins were resolved by SDS-polyacrylamide gel electrophoresis on $10 \%$ gels and transferred to nitrocellulose membranes (Amersham Biosciences). The membranes were probed with primary antibodies against GAPDH (1/20000, Sigma), PSD95 (1/12500, Sigma), and synaptophysin (1/15000, Sigma), blocked with $3 \%$ fish skin gelatin and then probed with IRDye $800 \mathrm{CW}$ goat anti-mouse, IRDye $680 \mathrm{CW}$ goat antirabbit secondary antibodies (LI-COR Biosciences). Blotted proteins were detected and quantified using the Odyssey infrared imaging system (LI-COR).

\section{Statistical analyses}

Data were analyzed using the InVivoStat software [20]. One-way analyses of variance (ANOVAs), two-way ANOVAs, and repeated measure ANOVAs, were followed by post-hoc planned comparisons when appropriate using Fisher PLSD test. $p<0.05$ was considered significant.

\section{RESULTS}

TASTPM mice show altered behavioral and HPA responses to novelty stress

We first quantified the acute novelty-induced rise in stress hormone levels in 4-month-old WT and TASTPM mice. The ACTH response of TASTPM mice was reduced but the corticosterone response was unaltered, despite elevated baseline plasma corticosterone levels (Supplementary Figure 1A, B; available online: http://www.j-alz.com/issues/34/vol34-3.html \#supplementarydata04). A blunted ACTH response associated with unaltered corticosterone response has also been seen in mild to moderate AD patients subjected to a CRF challenge [21], suggesting that CRF signaling is altered in pre-pathological TASTPM mice. We, therefore, investigated whether antagonism of CRFR1, using CP154,526, or CRFR2, using antisauvagine, altered neuroendocrine, physiological, and behavioral responses to acute novelty.
Novelty-induced ACTH release was attenuated by pre-treatment with either CRFR1 or CRFR2 antagonists in WT mice $(p=0.02, p=0.14$, and $p=0.24$ versus undisturbed levels, respectively, Fig. 1A). TASTPM' ACTH levels were unaltered by novelty exposure and CRFR1 or CRFR2 antagonism $(p=0.94, p=0.93$, and $p=0.33$ versus unexposed levels, respectively, Fig. 1A) but their basal $(p<0.001)$ and novelty-induced $(p=0.009)$ circulating corticosterone levels were higher than those of WT mice (Fig. 1B). Novelty induced corticosterone release in both WT $(p<0.001)$ and TASTPM $(p=0.005)$ mice, which was unaltered by CRFR1 or CRFR2 blockade in either genotype (all $p<0.001$ versus unexposed levels, Fig. 1B).

WT and TASTPM mice also differed markedly in their behavioral responses to novelty with TASTPM mice exhibiting less wall-leaning $\left(F_{(1,39)}=87.03\right.$; $p<0.001$, Fig. $1 C)$, rearing $\left(F_{(1,39)}=24.77 ; p<0.001\right.$, Fig. 1D), and grooming $\left(\mathrm{F}_{(1,39)}=6.95 ; p=0.01\right.$, Fig. 1E) behaviors, but more tail rattling $\left(F_{(1,39)}=\right.$ 27.16; $p<0.001$, Supplementary Figure 2A). CRFR1 antagonism significantly increased novelty-induced leaning ( $p=0.004)$ and rearing $(p=0.02)$ behaviors in WT, but not TASTPM mice $(p=0.74$ and $p=0.99$, respectively), consistent with reported anxiogenic effects of central CRFR1 activation [22]. CRFR2 antagonism failed to alter the behavioral responses of WT mice, but normalized grooming activity in TASTPM mice ( $p=0.63$ versus vehicle-treated WT, Fig. 1E). Thus, CRFR1 signaling is apparently reduced in TASTPM mice contributing to some aspects of their modified stress responses. CRFR1 is the main CRF receptor in the hippocampus and frontal cortex [23], two brain regions involved in contextual fear memory and extinction. We therefore investigated its involvement, via interactions with NMDAR, in the cognitive benefits of repeated novelty in TASTPM mice. The different stress responses of WT and TASTPM mice also prompted us to test the impact of repeated injection stress. Since this procedure produced adverse effects in WT but not TASTPM mice (detailed below), only TASTPM mice were used for the chronic pharmacological study.

After 5 weeks of novelty sessions, baseline corticosterone levels were no longer elevated in TASTPM mice and baseline ACTH levels did not differ, irrespective of the injection status (Supplementary Table 1), indicating that the early hypercorticolism of TASTPM mice was rescued by handling and exposing the mice to the novel cage. Antagonism of novelty-induced CRFR1 and/or NMDAR activation, using CP154,526 

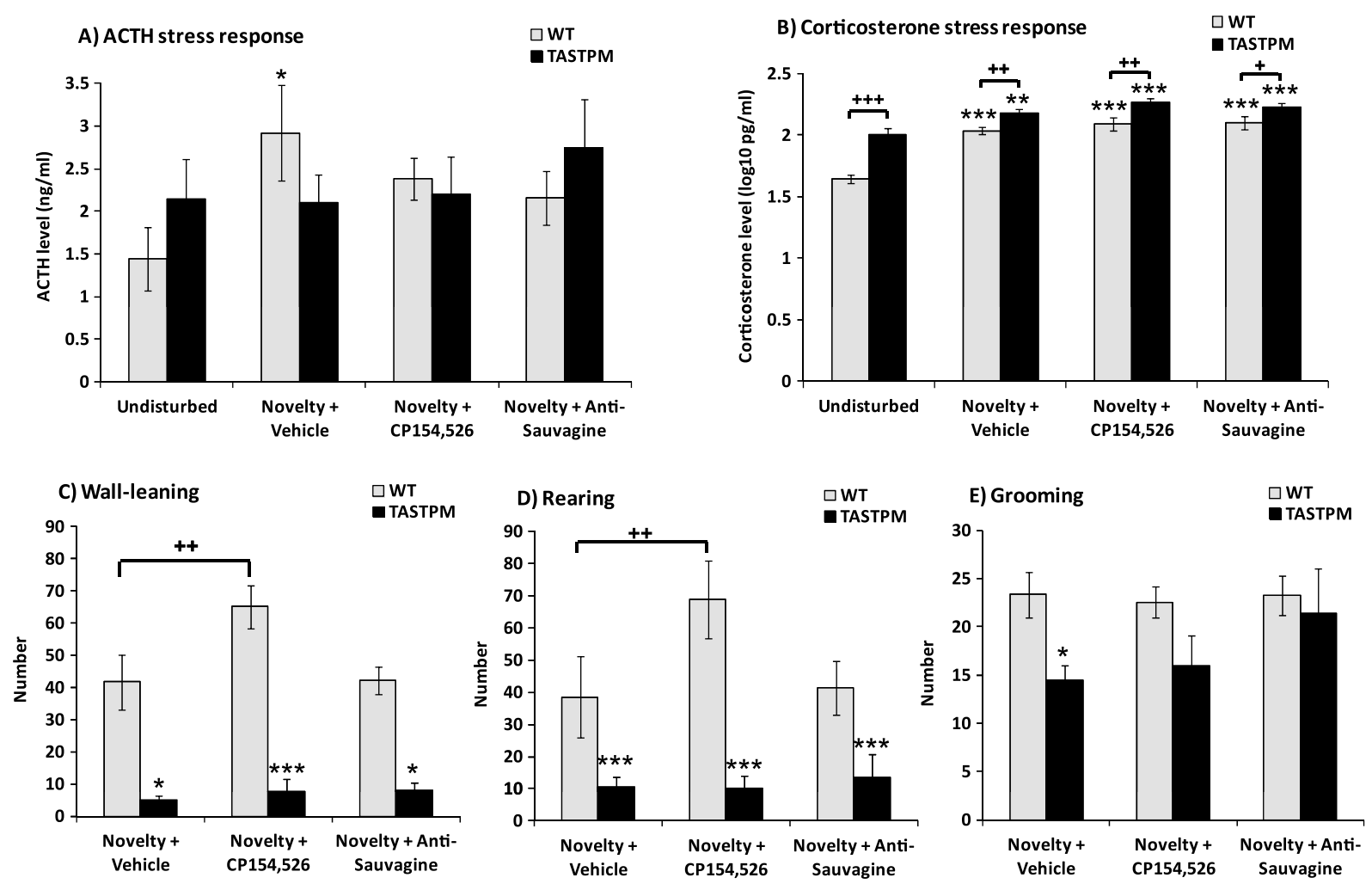

Fig. 1. Reduced CRFR1 signaling in TASTPM mice. WT and TASTPM mice were exposed to novelty for 1-hour, 30 minutes after receiving an i.c.v. injection of saline, CRFR1 or CRFR2 antagonists ( 7 nmol CP154,526 and 100 pmol anti-sauvagine, respectively). Novelty increased plasma ACTH levels in WT but not in TASTPM mice nor following CRFR1 and CRFR2 antagonism (A). TASTPM had increased baseline and novelty-induced levels of corticosterone, unaltered by CRFR1 or CRFR2 antagonism in either genotype (B). Blockade of novelty-induced CRFR1 activation increased wall-leaning (C) and rearing (D) behaviors in WT but not TASTPM mice, while CRFR2 blockade rescued noveltyinduced grooming levels in TASTPM mice (E). ${ }^{*} p<0.05,{ }^{* *} p<0.01,{ }^{* * *} p<0.001$ versus undisturbed (same genotype). ${ }^{+} p<0.05,{ }^{++} p<0.01$, ${ }^{+++} p<0.001$.

and MK801, respectively, did not alter baseline ACTH and corticosterone levels (Supplementary Table 2).

Defecations induced by acute novelty exposure were not altered in TASTPM mice or by prior antagonism of CRFR1 or CRFR2 in either genotype (Supplementary Figure 2B). Defecations induced by repeated novelty sessions in TASTPM mice were decreased by blocking CRFR1 activation and increased by blocking NMDAR activation $(p=0.03$ and $p=0.003$ versus vehicle-treated TASTPM), an effect attenuated by co-treatment with CP154,526 ( $p=0.16$ versus vehicle-treated TASTPM, Supplementary Figure 2C, Treatment: $\left.F_{(3,40)}=10.48 ; p<0.001\right)$. This is unlikely due to altered food intake as none of the drugs altered body weights (Supplementary Table 2). Thus, CRFR1 and NMDAR mediate opposing effects on physiological responses to novelty in TASTPM mice.

In summary, the altered stress responses of TASTPM mice were characterized by a reduced ACTH secre- tion and reduced vertical activity and self-grooming behavior as well as increased tail-rattling. The failure of CRFR1 antagonism to reduce the novelty-induced ACTH secretion and to increase vertical activity of TASTPM mice suggests that CRFR1 signaling is impaired in these mice contributing to their altered stress responsiveness.

CRFR1 activation contributes to the cognitive benefits of novelty exposure independently of NMDAR activation

Distances traveled in the open-field were unaltered by any of the experimental conditions (Supplementary Tables 1 and 2) indicating that differences in locomotor activity did not confound contextual fear conditioning, as assessed by immobility levels.

All experimental groups similarly acquired contextual fear (Fig. 2A, B). Contextual fear memory was 
Repeated i.p. injections in WT and TASTPM mice

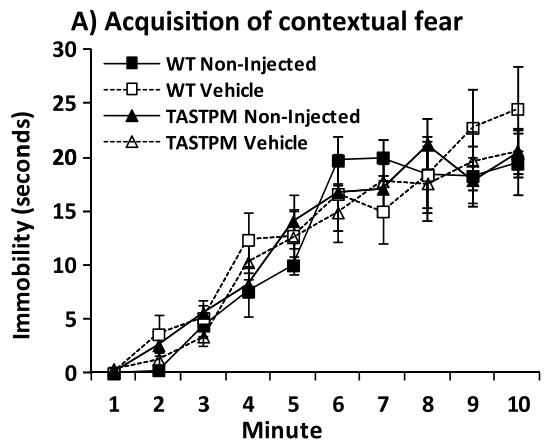

C) Contextual fear memory

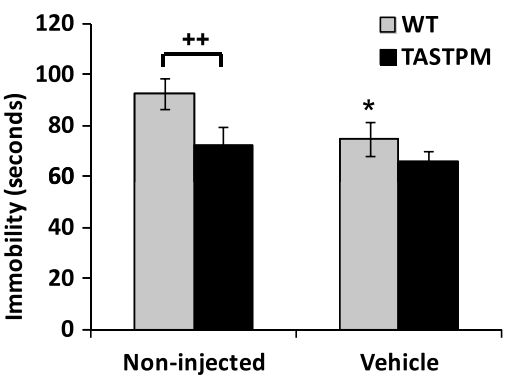

E) Contextual fear extinction

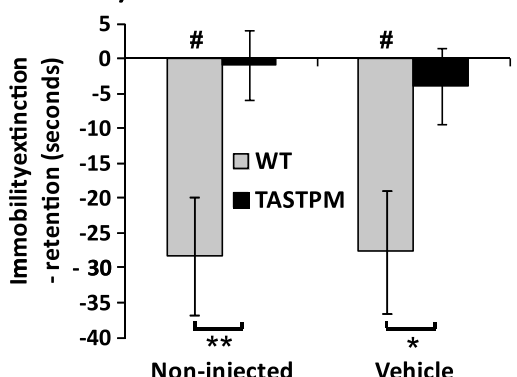

Non-injected

Vehicle

\section{Antagonism of repeated novelty-induced CRFR1 and/or NMDAR activation in TASTPM mice}
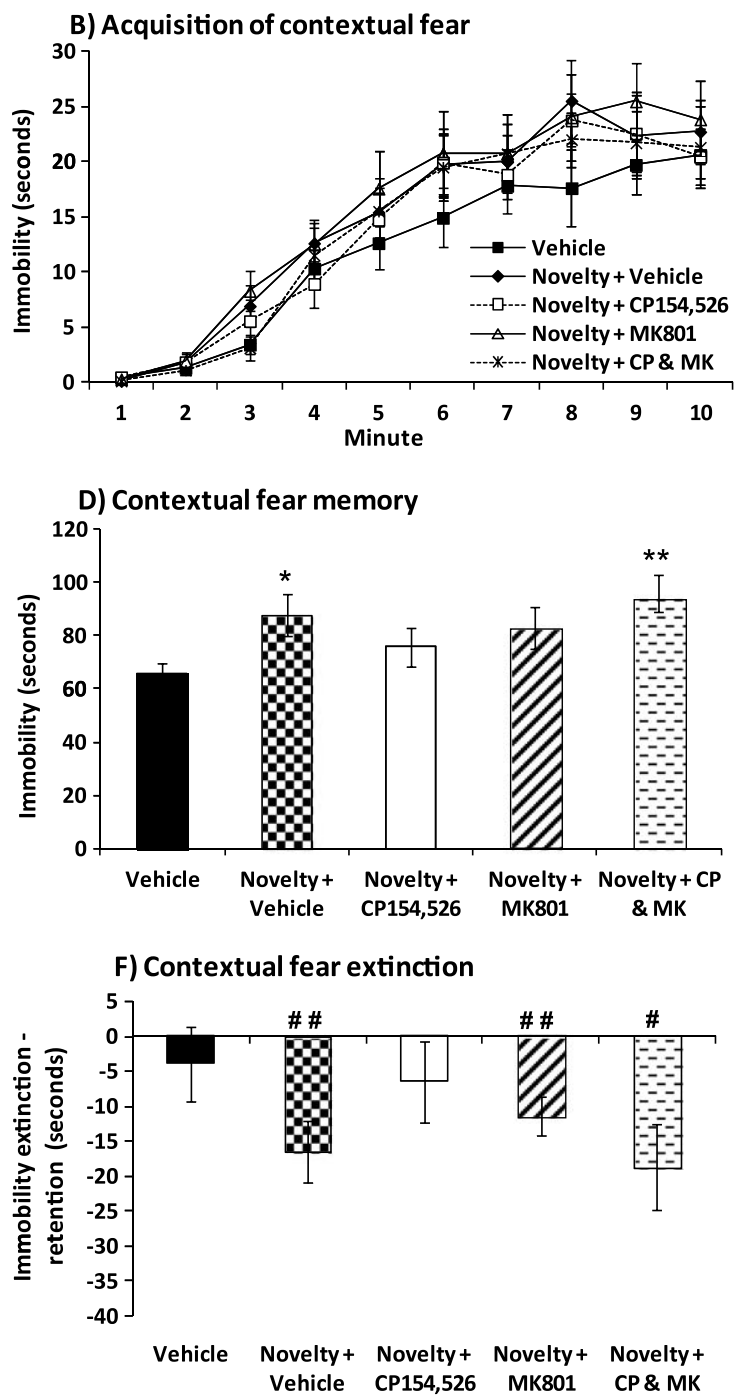

Fig. 2. CRFR1 signaling mediates novelty-induced cognitive improvement in TASTPM mice. After 20 novelty exposures combined with vehicle, CRFR1 and/or NMDAR antagonism (10 mg/kg CP154,526 and/or $0.1 \mathrm{mg} / \mathrm{kg}$ MK801, i.p.), mice were subjected to 10 context-footshock pairings. All groups similarly acquired contextual fear (A\& B) but fear memory was reduced in TASTPM mice and by the injection stress in WT but not TASTPM mice (C). Novelty exposures enhanced fear memory of TASTPM mice through CRFR1 and NMDAR activation independently of each other (D). Contextual fear extinction was impaired in TASTPM mice (E) and this was rescued by novelty-induced CRFR1 activation (F). ${ }^{*} p<0.05,{ }^{* *} p<0.01$ versus undisturbed vehicle-treated. ${ }^{\#} p<0.05,{ }^{\# \#} p<0.01$ versus 0 (lack ofextinction). ${ }^{++} p<0.01$.

lower in TASTPM mice $\left(F_{(1,31)}=5.26 ; p=0.03\right)$ and was reduced to TASTPM levels in vehicle-injected WT mice ( $p=0.32$ versus vehicle-treated TASTPM, Fig. 2C). Exposures to novelty enhanced fear memory of TASTPM mice ( $p=0.03$ versus unexposed vehicletreated), an effect prevented by CP154,526 and MK801 treatments $(p=0.32$ and $p=0.09$ versus unexposed vehicle-treated, respectively, Fig. 2D) suggesting that both CRFR1 and NMDAR activation contributed to the memory improvement, but independently, as a combination of antagonists did not prevent the memory enhancement $(p=0.004$ versus unexposed vehicletreated, Treatment: $F_{(4,47)}=2.63 ; p=0.04$, Fig. 2D).

Contextual fear extinction was reduced in TASTPM mice (Genotype: $F_{(1,31)}=14.16 ; p<0.001$ ) and, whereas WT mice showed significant memory 
Repeated i.p. injections in WT and TASTPM mice

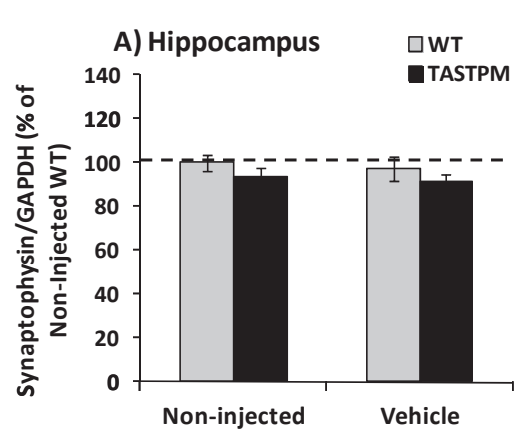

C) Frontal cortex $\square \mathrm{WT}$

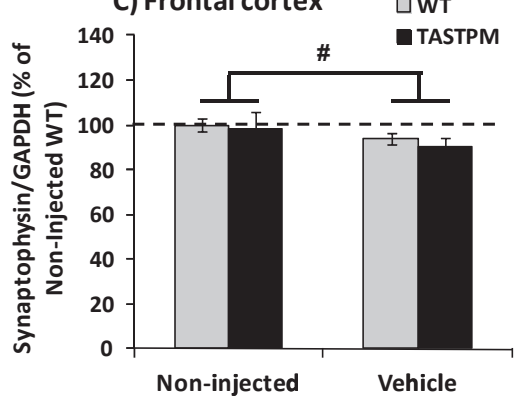

Antagonism of repeated novelty-induced CRFR1 and/or NMDAR activation in TASTPM mice

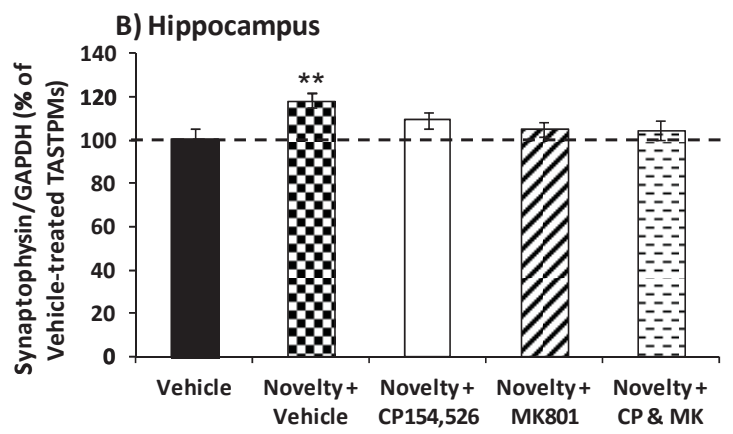

D) Frontal cortex

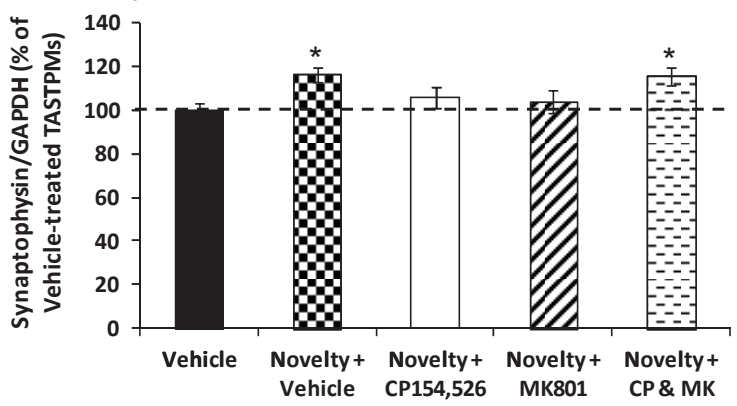

E) Representative western blots

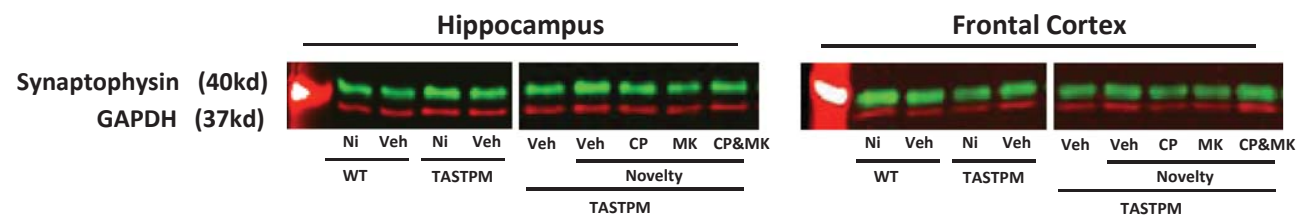

Fig. 3. CRFR1 signaling mediates the synaptogenic effects of novelty exposures. Hippocampal synaptophysin levels were unaltered in TASTPM mice (A) but were increased by repeated exposure to novelty via co-activation of CRFR1 and NMDAR (B). Fronto-cortical synaptophysin levels were reduced by repeated injections (C) but increased by novelty-induced CRFR1 and NMDAR activation independently of each other (D). Representative western blots (E). ${ }^{*} p<0.05,{ }^{* *} p<0.01$ versus undisturbed vehicle-treated. ${ }^{\#} p<0.05$.

extinction (non-injected $p=0.015$ and vehicle-treated $p=0.017$ versus 0 , i.e., lack of extinction), TASTPM did not (non-injected $p=0.86$ and vehicle-treated $p=0.48$ versus 0 , Fig. $2 \mathrm{E}$ ). The extinction deficit was rescued by repeated novelty sessions $(p=0.005$ versus 0 ), an effect prevented by antagonism of CRFR1 but not NMDAR alone or in combination with CRFR1 blockade ( $p=0.29, p=0.002$ and $p=0.014$ versus 0 , respectively, Fig. 2F).Thus, novelty-induced CRFR1 activation contributes to the memory improvement but not by directly activating NMDAR.

In summary, despite altered stress responses, TASTPM mice were resistant to the memory deficits caused by the stress of i.p. injections in WT mice. The repeated exposure of TASTPM mice to a novel environment increased contextual fear memory performance and reversed their deficit in contextual fear memory extinction via CRFR1 activation.

\section{CRFR1 activation mediates novelty-induced} increase in synaptic density region-dependently via NMDAR activation

Synaptophysin levels of WT and TASTPM mice did not differ in the hippocampus $\left(F_{(1,32)}=2.12\right.$; $p=0.15$, Fig. 3A) and frontal cortex $\left(F_{(1,32)}=0.49\right.$; 
Repeated i.p. injections in WT and TASTPM mice
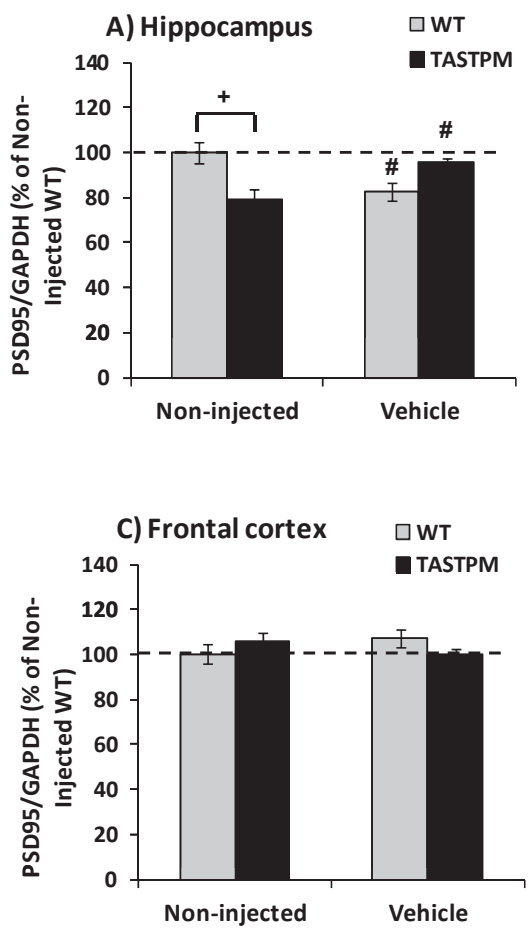

E) Representative western blots

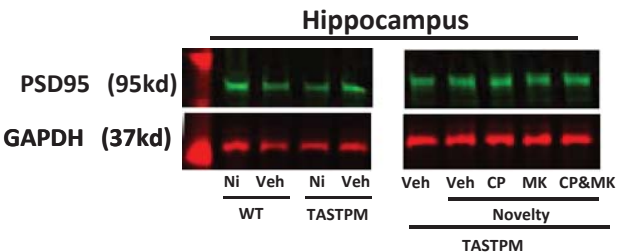

Antagonism of repeated novelty-induced CRFR1 and/or NMDAR activation in TASTPM mice
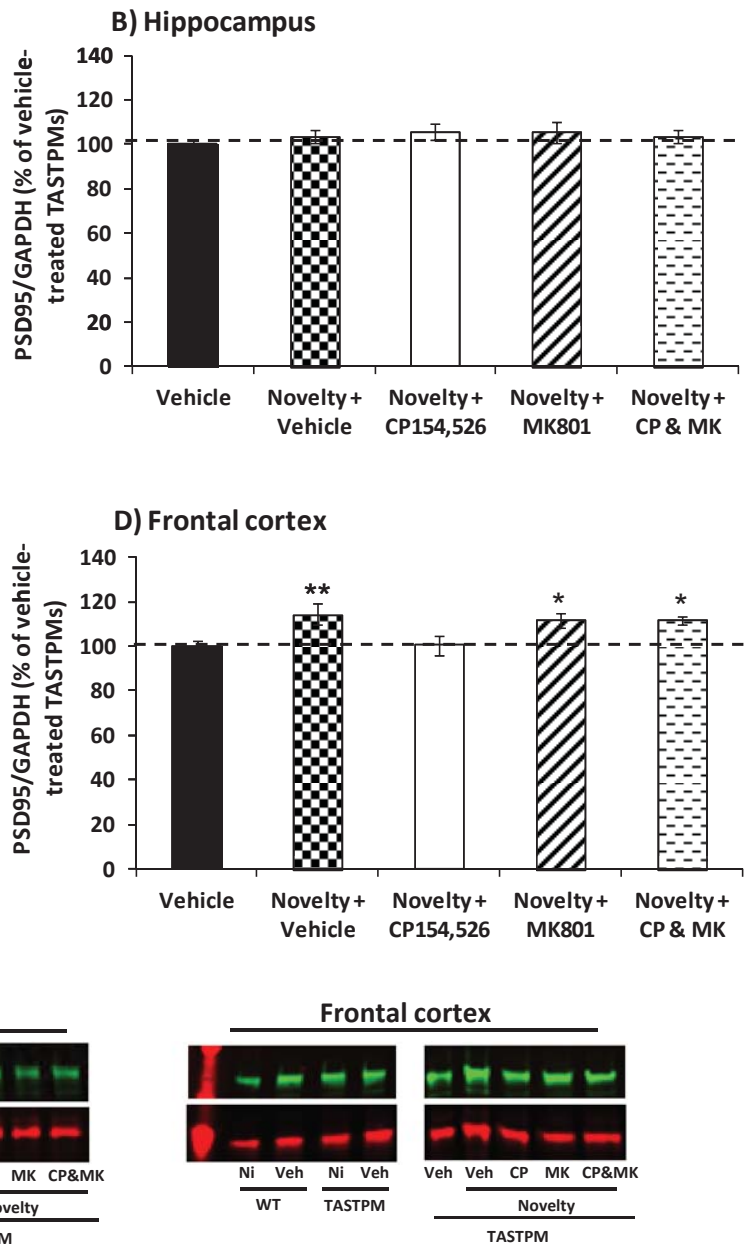

Fig. 4. Novelty-induced CRFR1 activation region-dependently enhances PSD95 levels. Hippocampal PSD95 levels were reduced in TASTPM mice; this was rescued by repeated injections which also reduced those levels in WT mice (A). Exposures to novelty did not alter hippocampal PSD95 levels (B). Fronto-cortical PSD95 levels were unaltered in TASTPM mice or by the injections (C) and were increased by novelty-induced CRFR1 but not NMDAR activation (D). Representative western blots (E). ${ }^{+} p<0.05$. ${ }^{\#} p<0.05$ versus non-injected. ${ }^{*} p<0.05,{ }^{* *} p<0.01$ versus undisturbed vehicle-treated. ${ }^{*} p<0.05$.

$p=0.49$, Fig. 3C) and were unaltered by an acute novelty session (Supplementary Figure 1C, D). Repeated injections reduced fronto-cortical $\left(F_{(1,32)}=4.17 ; p=0.05\right.$, Fig. 3C) but not hippocampal $\left(F_{(1,32)}=0.39 ; p=0.54\right.$, Fig. 3A) synaptophysin levels in both genotypes. Repeated novelty sessions increased synaptophysin levels of TASTPM mice in both brain regions ( $p=0.003$ and $p=0.01$ versus unexposed vehicle-treated for hippocampus and frontal cortex, respectively, Fig. 3B, D) via activation of CRFR1 and NMDAR (CP154,526 versus unexposed vehicle-treated $p=0.13$ and $p=0.33$; MK801 versus unexposed vehicle-treated $p=0.51$ and $p=0.41$ for hippocampus and frontal cortex, respectively, Fig. 3B, D). Antagonism of novelty-induced both CRFR1 and NMDAR activation prevented the rise in hippocampal but not fronto-cortical synaptophysin levels $(p=0.50$ and $p=0.01$ versus unexposed vehicle-treated, Fig. 3B, D). Thus, activation of both receptors is required for controlling synaptic density.

In contrast, PSD95 levels of TASTPM mice were reduced in the hippocampus (Supplementary Figure 1E 
Table 1

Summary of findings

\begin{tabular}{|c|c|c|}
\hline & Findings in TASTPM mice & Figure/Table \\
\hline \multirow[t]{3}{*}{ HPA axis activity } & Blunted ACTH stress response & Fig. 1A, Supp. Fig. 1A \\
\hline & CRFR1 antagonism failed to reduce ACTH secretion & Fig. 1A \\
\hline & Elevated baseline corticosterone levels & Fig. 1B, Supp. Fig. 1B \\
\hline \multirow[t]{4}{*}{ Behavioral stress response } & Reduced vertical activity and self-grooming behavior & Fig. 1C-E \\
\hline & Increased tail rattling behavior & Supp. Fig. 2A \\
\hline & CRFR1 antagonism failed to increase vertical activity & Fig. $1 C, D$ \\
\hline & CRFR2 antagonism restored self-grooming activity & Fig. 1E \\
\hline \multirow[t]{2}{*}{ Stress-induced defecations } & $\begin{array}{l}\text { Unaltered by acute novelty and CRFR1 or CRFR2 } \\
\text { antagonism }\end{array}$ & Supp. Fig. 1B \\
\hline & $\begin{array}{l}\text { Reduced by repeated CRFR1 antagonism and increased } \\
\text { by repeated NMDAR antagonism }\end{array}$ & Supp. Fig. 1C \\
\hline Locomotor activity & Unaltered by any treatment conditions & Supp. Tables 1 and 2 \\
\hline Acquisition of contextual fear memory & Unaltered by any treatment conditions & Fig. 2A, B \\
\hline \multirow{2}{*}{ Contextual fear memory } & Unaltered by the stress of repeated i.p. injections & Fig. $2 \mathrm{C}$ \\
\hline & $\begin{array}{l}\text { Improved by the repeated exposure to novelty via } \\
\text { CRFR1 activation }\end{array}$ & Fig. 2D \\
\hline \multirow{2}{*}{ Contextual fear extinction } & Impaired in TASTPM mice & Fig. 2E \\
\hline & $\begin{array}{l}\text { Rescued by the repeated exposure to novelty via CRFR } 1 \\
\text { activation }\end{array}$ & Fig. $2 \mathrm{~F}$ \\
\hline Synaptophysin levels & $\begin{array}{l}\text { Increased by the repeated exposure to novelty in both } \\
\text { the hippocampus and frontal cortex via CRFR } 1 \text { and } \\
\text { NMDAR activation }\end{array}$ & Fig. 3B, D \\
\hline \multirow[t]{2}{*}{ PSD95 levels } & $\begin{array}{l}\text { Reduced hippocampal levels rescued by the stress of } \\
\text { repeated i.p. injections }\end{array}$ & Fig. 4A, Supp. Fig. 1E \\
\hline & $\begin{array}{l}\text { Increased in the frontal cortex by the repeated exposure } \\
\text { to novelty via CRFR1 activation }\end{array}$ & Fig. 4D \\
\hline
\end{tabular}

and $p<0.001$ versus non-injected WT, Fig. 4A) but not frontal cortex (Supplementary Figure $1 \mathrm{~F}$ and $p=0.30$ versus non-injected WT, Fig. 4C), and unaltered by acute novelty (Supplementary Figure 1E, F). Repeated injections reduced hippocampal PSD95 levels of WT mice ( $p=0.004$ versus non-injected, Fig. 4A) but increased those of TASTPM mice $(p=0.002$ versus non-injected, Fig. 4A) thereby returning them to control levels ( $p=0.44$ versus non-injected WT, Fig. 4A). Neither repeated novelty, nor any of the drugs, altered hippocampal PSD95 levels (group: $F_{(4,47)}=0.44$; $p=0.78$, Fig. 4B). Fronto-cortical PSD95 levels of TASTPM mice were increased by repeated novelty sessions $(p=0.007$ versus unexposed vehicle-treated, Fig. 4D) via CRFR1 ( $p=0.94$ versus unexposed vehicle-treated, Fig. 4D) but not NMDAR activation as MK801 did not prevent this increase, either when administered alone or in combination with CP154,526 ( $p=0.02$ versus unexposed vehicle-treated in both cases, Fig. 4D). Thus, CRFR1 signaling during repeated exposures to novelty region-dependently modulates PSD95 levels.

In summary, at the age of 4 months, TASTPM mice exhibited reduced hippocampal levels of PSD95 and this was rescued by the repeated stress of injection. Repeated exposure to novelty stress enhanced synaptophysin levels in the hippocampus and frontal cortex of
TASTPM mice via activation of CRFR1 and NMDAR. Moreover, the increased in fronto-cortical PSD95 levels in TASTPM mice was mediated by activation of CRFR1 but not NMDAR.

\section{DISCUSSION}

The work reported here aimed to establish that a stimulating environment improved AD-like pathology by inducing a stress response and the key findings are summarized in Table 1 . We established here that CRFR1 signaling, a major regulator of neuroendocrine and behavioral stress responses, mediates the protective effects of a stimulating environment in TASTPM mice. We first showed that stress responses to a single exposure to a novel environment were altered in pre-pathological TASTPM mice with reduced CRFR1mediated effects, although they were resistant to the adverse cognitive and synaptic effects of repeated injection stress seen in WT mice. In contrast, repeated CRFR1 activation during the novelty sessions contributed to the improved contextual fear memory and extinction, and associated synaptogenesis, seen in TASTPM mice, indicating that the deficient CRFR1 responses were rescued by a stimulating environment, contributing to cognitive improvement. Although 
NMDAR activation critically modulates synaptic plasticity thought to underlie learning and memory [14], antagonism of novelty-induced NMDAR activation mimicked some of the effects of CRFR1 antagonism, but reversed rather than amplified its fronto-cortical cognitive and synaptic effects.

\section{CRFR1 signaling is impaired in early AD-like pathology in TASTPM mice, a mechanism for stress susceptibility?}

Despite epidemiological evidence linking premorbid stress susceptibility with an enhanced risk of AD [24], this has not been investigated in detail in mouse models. A prolonged corticosterone response to restraint stress, but unaltered baseline or stress-induced levels of the hormone have been reported in young A $\beta$ PP-overexpressing mice [25] while 3xTg AD mice, with mutated A $\beta P P$, PS1, and tau genes, have elevated baseline and stress-induced corticosterone levels [26]. The baseline hypercorticolism of asymptomatic TASTPM mice was associated with a blunted ACTH but an unaltered corticosterone response to acute novelty, and with a very distinct pattern of behavioral responses. A similar neuroendocrine profile has been observed in AD patients subjected to a CRF challenge [21], suggesting reduced CRF signaling. The failure of TASTPM mice to exhibit the behavioral and neuroendocrine effects of blockade of novelty-induced CRFR1 or CRFR2 activation further confirms this hypothesis. Whether this is a mechanism for stress susceptibility is unclear, as TASTPM mice were resistant to the adverse effects of repeated injection stress seen in WT mice. Injections induce a short-lasting corticosterone response [27], and thus, the altered HPA response of TASTPM mice may confer some protection against adverse effects of mild stressors. Indeed, the blunted ACTH stress response may prevent further HPA axis activation, consistent with the lack of differences between WT and TASTPM mice for stress levels of corticosterone, although later time points need to be investigated to confirm this hypothesis. Such stress resistance, however, is unlikely to protect against intense stressors since repeated restraint stress [28] and chronic mild stress [29] exacerbate AD-like pathology.

\section{CRFRI signaling contributes to AD progression}

We found that CRFR1 activation mediates the enhanced cognitive performance and synaptogenesis of TASTPM mice exposed to a stimulating environment, which is consistent with the hypothesis that reduced CRFR1 signaling contributes to human AD progression [9]. Moreover, social isolation stress upregulated hippocampal and cortical CRFR1 levels of A $\beta P P$ overexpressing mice [30], despite unaltered extracellular CRF levels [31], suggesting that chronic stress reduces CRFR1 signaling, thereby contributing to exacerbated AD-like pathology. Furthermore, CRF, via CRFR1, was found to protect cortical and hippocampal neurons from amyloid- $\beta$-induced death in vitro [32]. In contrast, increased $\mathrm{CRF}$ function was found to contribute to restraint stress-induced amyloid- $\beta$ release [31] and exacerbation of plaque load [28] in A $\beta P P$ and A $\beta P P / P S 1$ overexpressing mice, respectively, whereas $\mathrm{A} \beta P P$ transgenic mice overexpressing CRF displayed exacerbated cognitive and amyloid pathology [33]. This further supports a role for $\mathrm{CRF}$ in $\mathrm{AD}$ progression, although the receptor(s) mediating these effects need to be established. CRF dose-dependently increases amyloid- $\beta$ release [31], leading to the hypothesis that it produces positive or negative effects on $\mathrm{AD}$ progression as function of the severity of the stressors, although a CRF receptor-dependency cannot be excluded. Indeed, CRFR2, but not CRFR1 deletion, was found to mediate stress-induced tau phosphorylation in wild-type mice [34]. Moreover, CRFR2 antagonism rescued noveltyinduced grooming levels in TASTPM mice suggesting that its altered function also contributes to AD progression. The lack of a selective CRFR2 antagonist that crosses the blood-brain barrier, however, prevented us from testing its involvement in the protective effects of repeated novelty.

\section{Synaptogenesis contributes to novelty-induced cognitive improvement in a CRFRl-and NMDAR-dependent manner}

CRF, via CRFR1, modulates hippocampal synaptic function and plasticity and, thereby, learning and memory, in a time- and dose-dependent manner, with short exposure to stress levels promoting positive effects but long exposure promoting synapse loss and cognitive impairments [12]. Thus, the blockade of noveltyinduced increases in synaptophysin levels by CRFR1 antagonism suggests that the improved contextual fear memory and extinction, which require hippocampal and fronto-cortical integrity, are mediated by an increase in synaptic density resulting from repeated, intermittent CRF secretion. Our data also suggest that NMDAR activation region-dependently contributes to the synaptogenic and cognition-enhancing effects of novelty sessions. In support of this hypothesis, 
NMDAR activation was found to alter synaptogenesis in vitro, in a time-dependent manner, promoting it in the short-term and reducing it as a result of chronic activation [35]. In vivo, pre-treatment with MK801 was found to prevent water-maze overtraining-induced synaptogenesis in the adult hippocampus and impaired memory performance [36] while, in a genetic model of NMDAR hypofunction, memory deficits and reduced fronto-cortical dendritic spine density were observed [37]. Co-antagonism of CRFR1 and NMDAR, however, prevented the rise in hippocampal, but not fronto-cortical, synaptophysin levels, suggesting that the two receptors stimulate each other in the former, but not latter, brain area to induce protective effects. This is consistent with the reported region-dependent modulation of glutamatergic transmission by $\mathrm{CRF}$, which, via CRFR1, was found to depress glutamatergic transmission in the central nucleus of the amygdala, while facilitating it in the lateral septum mediolateral nucleus [13], hippocampus and basolateral amygdala [22]. In rat fronto-cortical slices, however, CRF was found to inhibit glutamatergic synaptic transmission in a NMDAR-dependent manner [38]. Thus, the enhanced fronto-cortical synaptic density and extinction of contextual fear following blockade of novelty-induced both CRFR1 and NMDAR activation likely reflects synergistic facilitatory effects on synaptic glutamatergic transmission. Altogether, this suggests that CRFR1 activation protects against $\mathrm{AD}$-related impairment by inducing synaptogenesis through directly enhancing hippocampal, but not fronto-cortical NMDAR function.

\section{Novelty-induced CRFRI activation enhances fronto-cortical PSD95 levels}

PSD95 is an activity-dependent regulator of NMDAR and synaptic plasticity and is thought to act as a sensor regulating MMDAR function in response to alterations in synaptic activity [39]. Our results, showing that hippocampal PSD95 levels are reduced in 4-month-old TASTPM mice, suggest that functional synaptic plasticity of the hippocampus is impaired prior to the onset of behavioral symptoms. This reduction in PSD95 levels was still evident at 5.5 months, once contextual fear memory was impaired. The injection stress reversed the reduced hippocampal PSD95 levels of TASTPM mice but not the memory deficits while decreasing memory performance and hippocampal PSD95 levels of WT mice, suggesting that other molecular mechanisms also contribute to AD-related cognitive symptoms. PSD95 levels are closely linked

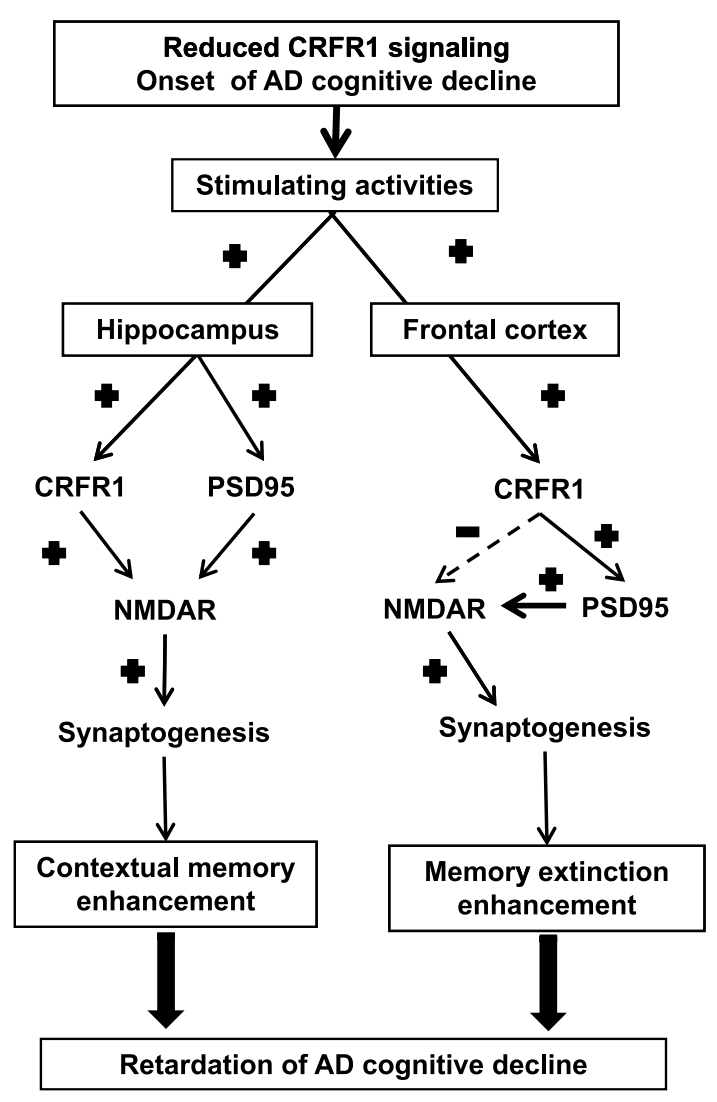

Fig. 5. Hypothetical mechanism underling protective effects of positive lifestyle factors on the progression of Alzheimer's disease (AD) cognitive decline. The deficient CRFR1 signaling at the onset of cognitive symptoms would be rescued by an active lifestyle resulting in enhanced cognitive function through directly increasing NMDARmediated synaptic plasticity and synaptogenesis in the hippocampus, and indirectly in the frontal cortex via accumulation of PSD95.

to the function of glutamatergic receptors, potentiating activation of NMDAR through direct and indirect interactions. PSD95 accumulation accelerates the maturation of excitatory synapses [40], increases NMDAR protein levels [39] and reduces NMDAR desensitization [41]. Thus, we hypothesize that the reduced hippocampal PSD95 levels of young TASTPM mice associated with unaltered glutamate levels (Supplementary Figure 1G) reflect NMDAR hypofunction, the rescue of which by repeated injections enabled the beneficial effects of CRFR1 activation on hippocampal synaptogenesis and function. In the frontal cortex, the enhanced PSD95 level was prevented by blockade of novelty-induced CRFR1 activation. This suggests that CRFR1 indirectly stimulates NMDAR function through an increase in PSD95 levels possibly occurring to compensate for CRF-induced depression of synaptic glutamatergic transmission. The failure of 
the combination of antagonists to prevent the noveltyinduced increase in fronto-cortical PSD95 levels, would therefore be the consequence of the antagonistic effects of CRFR1 and NMDAR activation, the former directly inhibiting and the latter stimulating synaptic glutamatergic transmission. Further studies are, however, needed to verify this hypothesis. Our data also further support the observation that genetically induced NMDAR hypofunction does not alter PSD95 levels in the mouse hippocampus or frontal cortex [42], but to the best of our knowledge, this is the first evidence that CRFR1 activation increases PSD95 levels. This may be a mechanism whereby CRF indirectly potentiates NMDAR-related synaptic plasticity and synaptogenesis.

\section{CONCLUSIONS}

The data presented in this paper suggest a novel mechanism whereby a stimulating environment can delay the progression of AD-like symptoms and reverse the pre-morbid stress susceptibility contributing to the disease. Accordingly, the intermittent secretion of CRF via CRFR1 facilitates NMDARmediated synaptic plasticity and synaptogenesis which are critical for learning and memory performance either directly or indirectly by promoting accumulation of PSD95 (Fig. 5).

\section{ACKNOWLEDGMENTS}

This work was supported by a Research Into Ageing New Investigator research grant (RIA-316) and RCUK fellowship to M-C.P. Thanks to GlaxoSmithKline Neurology CEDD for providing the mice and Prof. DA Kendall for helpful comments of the manuscript.

Authors' disclosures available online (http://www.jalz.com/disclosures/view.php?id=1603).

\section{REFERENCES}

[1] Pardon MC (2010) Therapeutic potential of some stress mediators in early Alzheimer's disease. Exp Gerontol 46, 170-173.

[2] Paillard-Borg S, Fratiglioni L, Xu W, Winblad B, Wang HX (2012) An active lifestyle postpones dementia onset by more than one year in very old adults. J Alzheimers Dis 31, 835-842.

[3] Hall CB, Lipton RB, Sliwinski M, Katz MJ, Derby CA, Verghese J (2009) Cognitive activities delay onset of memory decline in persons who develop dementia. Neurology $\mathbf{7 3}$, 356-361.

[4] Pardon MC, Sarmad S, Rattray I, Bates TE, Scullion GA, Marsden CA, Barrett DA, Lowe J, Kendall DA (2009) Repeated novel cage exposure-induced improvement of early Alzheimer's-like cognitive and amyloid changes in TASTPM mice is unrelated to changes in brain endocannabinoids levels. Neurobiol Aging 30, 1099-10113.

[5] Pardon M-C, Kendall DA, Perez-Diaz F, Duxon MS, Marsden CA (2004) Repeated sensory contact with aggressive mice rapidly leads to an anticipatory increase in core body temperature and physical activity that precedes the onset of aversive responding. Eur J Neurosci 20, 1033-1050.

[6] Rattray I, Pitiot A, Lowe J, Auer DP, Lima SJ, Schubert MI, Prior MJ, Marsden CA, Diaz FP, Kendall DA, Pardon MC (2010) Novel cage stress alters remote contextual fear extinction and regional T2 magnetic resonance relaxation times in TASTPM mice overexpressing amyloid. J Alzheimers Dis $\mathbf{2 0}$, 1049-1068.

[7] Bale TL, Vale WW (2004) CRF and CRF receptors: Role in stress responsivity and other behaviors. Annu Rev Pharmacol Toxicol 44, 525-557.

[8] Dube T, Brunson T, Nehlig A, Baram TZ (2000) Activation of specific neuronal circuits by corticotropin releasing hormone as indicated by c-fos expression and glucose metabolism. J Cereb Blood Flow Metab 20, 1414-1424.

[9] Bayatti N, Behl C (2005) The neuroprotective actions of corticotropin releasing hormone. Ageing Res Rev 4, 258-270.

[10] Souza-Talarico JN, Caramelli P, Nitrini R, Chaves EC (2008) Effect of cortisol levels on working memory performance in elderly subjects with Alzheimer's disease. Arq Neuropsiquiatr 66, 619-624.

[11] Terry RD, Masliah E, Salmon DP, Butters N, DeTeresa R, Hill R, Hansen LA, Katzman R (1991) Physical basis of cognitive alterations in Alzheimer's disease: Synapse loss is the major correlate of cognitive impairment. Ann Neurol 30, 572580.

[12] Chen Y, Andres AL, Frotscher M, Baram TZ (2012) Tuning synaptic transmission in the hippocampus by stress: The CRH system. Front Cell Neurosci $\mathbf{6}, 13$.

[13] Liu J, Yu B, Neugebauer V, Grigoriadis DE, Rivier J, Vale WW, Shinnick-Gallagher P, Gallagher JP (2004) Corticotropin-releasing factor and Urocortin I modulate excitatory glutamatergic synaptic transmission. J Neurosci 24, 4020-4029.

[14] Zorumski CF, Izumi Y (2012) NMDA receptors and metaplasticity: Mechanisms and possible roles in neuropsychiatric disorders. Neurosci Biobehav Rev 36, 989-1000.

[15] Howlett DR, Richardson JC, Austin A, Parsons AA, Bate ST, Davies DC, Gonzalez MI (2004) Cognitive correlates of Abeta deposition in male and female mice bearing amyloid precursor protein and presenilin-1 mutant transgenes. Brain Res 1017, 130-136.

[16] Rattray I, Scullion GA, Soulby A, Kendall DA, Pardon MC (2009) The occurrence of a deficit in contextual fear extinction in adult amyloid-over-expressing TASTPM mice is independent of the strength of conditioning but can be prevented by mild novel cage stress. Behav Brain Res 200, 83-90.

[17] Scullion GA, Kendall DA, Sunter D, Marsden CA, Pardon MC (2009) Central noradrenergic depletion by DSP-4 prevents stress-induced memory impairments in the object recognition task. Neuroscience 164, 415-423.

[18] Scullion GA, Kendall DA, Marsden CA, Sunter D, Pardon MC (2011) Chronic treatment with the alpha(2)-adrenoceptor antagonist fluparoxan prevents age-related deficits in spatial working memory in APPxPS1 transgenic mice without altering beta-amyloid plaque load or astrocytosis. Neuropharmacology 60, 223-234.

[19] Pardon MC, Roberts RE, Marsden CA, Bianchi M, Latif ML, Duxon MS, Kendall DA (2005) Social threat and novel cage stress-induced sustained extracellular-regulated 
kinase1/2 (ERK1/2) phosphorylation but differential modulation of brain-derived neurotrophic factor (BDNF) expression in the hippocampus of NMRI mice. Neuroscience 132, 561574.

[20] Clark RA, Shoaib M, Hewitt KN, Stanford SC, Bate ST (2012) A comparison of InVivoStat with other statistical software packages for analysis of data generated from animal experiments. J Psychopharmacol 26, 1136-1142.

[21] Nasman B, Olsson T, Fagerlund M, Eriksson S, Viitanen M, Carlstrom K (1996) Blunted adrenocorticotropin and increased adrenal steroid response to human corticotropinreleasing hormone in Alzheimer's disease. Biol Psychiatry 39, 311-318.

[22] Refojo D, Schweizer M, Kuehne C, Ehrenberg S, Thoeringer C, Vogl AM, Dedic N, Schumacher M, von Wolff G, Avrabos C, Touma C, Engblom D, Schutz G, Nave KA, Eder M, Wotjak CT, Sillaber I, Holsboer F, Wurst W, Deussing JM (2011) Glutamatergic and dopaminergic neurons mediate anxiogenic and anxiolytic effects of CRHR1. Science 333, 19031907.

[23] Chalmers DT, Lovenberg TW, De Souza EB (1995) Localization of novel corticotropin-releasing factor receptor (CRF2) mRNA expression to specific subcortical nuclei in rat brain: Comparison with CRF1 receptor mRNA expression. $\mathrm{J} \mathrm{Neu}$ rosci 15, 6340-6350.

[24] Wilson RS, Fleischman DA, Myers RA, Bennett DA, Bienias JL, Gilley DW, Evans DA (2004) Premorbid proneness to distress and episodic memory impairment in Alzheimer's disease. J Neurol Neurosurg Psychiatry 75, 191-195.

[25] Pedersen WA, Culmsee C, Ziegler D, Herman JP, Mattson MP (1999) Aberrant stress response associated with severe hypoglycemia in a transgenic mouse model of Alzheimer's disease. J Mol Neurosci 13, 159-165.

[26] Rothman SM, Herdener N, Camandola S, Texel SJ, Mughal MR, Cong WN, Martin B, Mattson MP (2012) 3xTgAD mice exhibit altered behavior and elevated Abeta after chronic mild social stress. Neurobiol Aging 33, 830 e1-e12.

[27] Meijer MK, Lemmens AG, Van Zutphen BF, Baumans V (2005) Urinary corticosterone levels in mice in response to intraperitoneal injections with saline. J Appl Anim Welf Sci $\mathbf{8}$, 279-283.

[28] Seo JS, Lee KW, Kim TK, Baek IS, Im JY, Han PL (2011) Behavioral stress causes mitochondrial dysfunction via $\mathrm{ABAD}$ up-regulation and aggravates plaque pathology in the brain of a mouse model of Alzheimer disease. Free Radic Biol Med 50, 1526-1535.

[29] Cuadrado-Tejedor M, Ricobaraza A, Frechilla D, Franco R, Perez-Mediavilla A, Garcia-Osta A (2012) Chronic mild stress accelerates the onset and progression of the Alzheimer's disease phenotype in Tg2576 mice. J Alzheimers Dis 28, 567 578.

[30] Dong H, Yuede CM, Yoo HS, Martin MV, Deal C, Mace AG, Csernansky JG (2008) Corticosterone and related receptor expression are associated with increased betaamyloid plaques in isolated Tg2576 mice. Neuroscience $\mathbf{1 5 5}$, 154-163.

[31] Kang JE, Cirrito JR, Dong H, Csernansky JG, Holtzman DM (2007) Acute stress increases interstitial fluid amyloid-beta via corticotropin-releasing factor and neuronal activity. Proc Natl Acad Sci U S A 104, 10673-10678.

[32] Facci L, Stevens DA, Pangallo M, Franceschini D, Skaper SD, Strijbos PJ (2003) Corticotropin-releasing factor (CRF) and related peptides confer neuroprotection via type $1 \mathrm{CRF}$ receptors. Neuropharmacology 45, 623-636.

[33] Dong H, Murphy KM, Meng L, Montalvo-Ortiz J, Zeng Z, Kolber BJ, Zhang S, Muglia LJ, Csernansky JG (2012) Corticotrophin releasing factor accelerates neuropathology and cognitive decline in a mouse model of Alzheimer's disease. J Alzheimers Dis 28, 579-592.

[34] Rissman RA, Staup MA, Lee AR, Justice NJ, Rice KC, Vale W, Sawchenko PE (2012) Corticotropin-releasing factor receptor-dependent effects of repeated stress on tau phosphorylation, solubility, and aggregation. Proc Natl Acad Sci U S A 109, 6277-6282.

[35] Punnoose A, Miskevich F, Townsend M, Constantine-Paton M (2004) The effects of NMDA receptor activation on synaptogenesis. MURJ 20, 48-51.

[36] Ramirez-Amaya V, Balderas I, Sandoval J, Escobar ML, Bermudez-Rattoni F (2001) Spatial long-term memory is related to mossy fiber synaptogenesis. J Neurosci 21, 73407348.

[37] DeVito LM, Balu DT, Kanter BR, Lykken C, Basu AC, Coyle JT, Eichenbaum H (2011) Serine racemase deletion disrupts memory for order and alters cortical dendritic morphology. Genes Brain Behav 10, 210-222.

[38] Zieba B, Grzegorzewska M, Branski P, Domin H, Wieronska JM, Hess G, Smialowska M (2008) The behavioural and electrophysiological effects of CRF in rat frontal cortex. Neuropeptides 42, 513-523.

[39] Lin Y, Skeberdis VA, Francesconi A, Bennett MV, Zukin RS (2004) Postsynaptic density protein-95 regulates NMDA channel gating and surface expression. J Neurosci 24, 1013810148.

[40] El-Husseini AE, Schnell E, Chetkovich DM, Nicoll RA, Bredt DS (2000) PSD-95 involvement in maturation of excitatory synapses. Science 290, 1364-1368.

[41] Li B, Otsu Y, Murphy TH, Raymond LA (2003) Developmental decrease in NMDA receptor desensitization associated with shift to synapse and interaction with postsynaptic density-95. J Neurosci 23, 11244-11254.

[42] Balu DT, Coyle JT (2011) Glutamate receptor composition of the post-synaptic density is altered in genetic mouse models of NMDA receptor hypo- and hyperfunction. Brain Res $\mathbf{1 3 9 2 ,}$ $1-7$. 